\title{
Prioridade para futuras vacinações contra a Covid-19 no Brasil: os usuários de transporte público devem ser um grupo-alvo?
}

\author{
Douglas Sathler \\ Guilherme de Castro Leiva**
}

\begin{abstract}
Considerando o recente surgimento de vacinas eficazes contra a Covid-19 e os escassos recursos para atender imediatamente à maior parte da população mundial, as sociedades precisam definir a ordem em que os grupos de cidadãos terão acesso às novas vacinas. As campanhas de vacinação devem priorizar a imunização de indivíduos vulneráveis e encarregados de ajudar outras pessoas, reduzindo as perdas humanas e minimizando os danos sociais e econômicos. No Brasil, os usuários de transporte público nas grandes cidades apresentam altos níveis de vulnerabilidade, diante de fatores relacionados à configuração espacial, à organização dos sistemas de transporte e ao alto percentual de pessoas de baixo nível socioeconômico em grandes periferias que dependem exclusivamente do transporte público para acesso a empregos e serviços básicos. A imunização dos usuários do transporte público pode produzir efeitos práticos relevantes no combate à Covid-19 no Brasil, tais como economia de recursos públicos, redução do número de óbitos e maior eficiência no controle setorizado da doença nas cidades. Portanto, sugerimos que os formuladores de políticas devem considerar os usuários frequentes de transporte público das grandes cidades brasileiras como um grupo-alvo nas campanhas de vacinação, dando a esse grupo um certo nível de prioridade com base em um mapeamento de risco adequado em nível local.
\end{abstract}

Palavras-chave: Vacina. Covid-19. Transporte público. Grupo-alvo.

\footnotetext{
* Faculdade Interdisciplinar de Humanidades (FIH), Centro de Geociências (Cegeo), Universidade Federal dos Vales do Jequitinhonha e Mucuri (UFVJM), Diamantina-MG, Brasil (doug.sathler@gmail.com; https://orcid.org/0000-0002-1547-5522).

** Departamento de Engenharia de Transportes (DET), Centro Federal de Educação Tecnológica de Minas Gerais (Cefet-MG), Belo Horizonte-MG, Brasil (guilherme.leiva@cefetmg.br; https://orcid.org/0000-0002-9228-1908).
} 


\section{Introdução}

A pandemia de Covid-19 requer soluções rápidas e eficazes em todo o mundo, colocando governos, legisladores, setores privados e cientistas sob enorme pressão. 0 desenvolvimento de vacinas parece ser o meio mais promissor de lidar adequadamente com essa crise de saúde. A gama de plataformas de tecnologia e abordagens consideradas aumentou as chances de sucesso no desenvolvimento de vacinas, como ácido nucleico, partícula semelhante a vírus, peptídeo, vetor viral, proteína recombinante, vírus vivo atenuado e inativado (THANH LE et al., 2020). Dado o recente surgimento de vacinas eficazes, os efeitos negativos da pandemia podem ser minimizados com a otimização do processo de vacinação em massa, não apenas alcançando uma taxa de vacinação suficientemente alta e rápida, mas também selecionando grupos prioritários de forma inteligente.

Nos países mais afetados pela pandemia, não há consenso na arena política sobre quem deve ter acesso às vacinas primeiro. No relatório denominado Ética e Covid-19: alocação de recursos e definição de prioridades, a Organização Mundial da Saúde (OMS) destaca que os principais princípios éticos para decidir quais grupos podem ser priorizados em um contexto de recursos escassos são igualdade e melhores resultados (WHO, 2020). Este relatório também sugere que a futura vacinação contra a Covid-19 deve priorizar pessoas com fatores de maior risco, como indivíduos com comorbidades (doenças cardiovasculares, obesidade, diabetes e hipertensão), populações idosas e marginalizadas, bem como trabalhadores de atividades essenciais (saúde e saneamento, setor alimentar e pessoal de transporte público).

No Brasil, dados do Ministério da Saúde revelam que o novo coronavírus (Sars-CoV-2) tem se disseminado rapidamente no país, principalmente nos grandes centros urbanos. Em 4 de junho de 2021, o país atingiu 469.388 óbitos e 16,8 milhões de casos registrados, o que corresponde a $12,69 \%$ e $9,78 \%$ dos registros mundiais, respectivamente. Na mesma data, Rio de Janeiro (26.667), São Paulo (30.978), Manaus (8.993), Brasília (8.740), Fortaleza (8.569), Salvador (6.737) e Recife (4.317) apresentavam os maiores números de óbitos entre as cidades do país (BRASIL, 2021). Além disso, Belo Horizonte, município-núcleo da terceira maior região metropolitana do Brasil, registrou 5.115 mortes. Esses dados epidemiológicos preocupantes criam as condições necessárias para o teste de novas vacinas no Brasil. De fato, o país estabeleceu parcerias de transferência de tecnologia para teste de vacinas, articulando centros nacionais consolidados (como Fiocruz e Butantã) com instituições e empresas estrangeiras, como Oxford-AstraZenica (Reino Unido) e Sinovac (China). Apesar do sucesso dessas parcerias no desenvolvimento de vacinas, a campanha de vacinação no Brasil está significativamente atrasada. 0 país registrou 47.636 .424 milhões de pessoas que tiveram acesso à primeira dose das vacinas até 4 de junho de 2021, o que corresponde a $22,3 \%$ da população total. Nesta data, o Brasil era o $64^{\circ}$ país do mundo no ranking global de população imunizada por 100 habitantes. 
O plano de vacinação brasileiro, recentemente, incluiu 77,2 milhões de pessoas no grupo prioritário, considerando apenas duas categorias principais:

- pessoas com 60 anos ou mais institucionalizadas, pessoas institucionalizadas com deficiência, povos indígenas que vivem em terras indígenas, trabalhadores da saúde;

- pessoas com 75 anos ou mais, povos e comunidades ribeirinhas tradicionais, povos e comunidades quilombolas tradicionais, pessoas de 60 a 74 anos, pessoas com comorbidades, pessoas com deficiências permanentes graves, moradores de rua, presidiários, funcionários da prisão, trabalhadores da educação, forças de segurança e resgate, militares, trabalhadores do transporte (incluindo caminhoneiros), trabalhadores portuários, trabalhadores da indústria (BRASIL, 2021).

Os epidemiologistas têm criticado estes grupos prioritários no plano nacional de vacinação, especialmente a recente inclusão de caminhoneiros e trabalhadores da indústria (DIAS, 2020). Se o grupo de prioridade for enorme, não haverá prioridade real. Além disso, não está claro qual será a prioridade da vacinação ao finalizar a imunização dessas 77,2 milhões de pessoas. A definição dessa ordem de prioridade também é essencial para 0 planejamento de ações, como o registro de grupos específicos de vacinação.

\section{Prioridades futuras para vacinação contra a Covid-19: os usuários de transporte público}

Após vacinar esses dois grupos definidos, sugerimos que as autoridades brasileiras, ao decidirem sobre a prioridade de vacinação, também considerem os usuários de transporte público nas grandes cidades, diante dos riscos inerentes associados à aglomeração de pessoas no transporte público, da alta vulnerabilidade social da maioria dos usuários e das falhas significativas no financiamento do transporte público no Brasil.

De acordo com Zhen et al. (2020), o desincentivo ou proibição do uso do transporte público está presente na maioria das diretrizes internacionais. No entanto, as autoridades precisam garantir que o transporte público seja capaz de suportar o funcionamento das atividades essenciais nas cidades (BARCELOS; BLANK, 2020; SILVA et al., 2020). Essas recomendações baseiam-se no consenso de que existe uma forte relação entre a propagação de doenças virais e o funcionamento do transporte público (MATEUS et al., 2014; SILVA et al., 2020). A Organização para a Cooperação e Desenvolvimento Econômico (OCDE) argumenta que o transporte público tem afetado significativamente a disseminação de Covid-19 nas cidades e redes urbanas (ECDC, 2020). De acordo com o recente relatório divulgado pelo Centro Europeu de Prevenção e Controle de Doenças (ECDC, 2020), a aglomeração no transporte público pode aumentar a transmissão direta (gotas respiratórias) e indireta (superfícies contaminadas) de Covid-19 entre usuários e funcionários. Além disso, Pardo et al. (2021) mostram que a alta densidade e níveis de mistura de pessoas dentro de ônibus e metrôs podem converter o sistema de transporte público em locais propensos a disseminar 
a Covid-19. Segundo os autores, a operação segura do transporte público deve contemplar alguns fatores-chave, como ventilação, comportamento do usuário (máscaras e proteção ocular), silêncio do usuário e distância entre passageiros (1-2 metros). Porém, nem sempre todos esses fatores são adotados prontamente, o que torna o transporte público um importante local de disseminação da Covid-19 (PARDO et al., 2021).

De fato, estudos recentes confirmam os altos riscos que envolvem o transporte público nesta pandemia. Zheng et al. (2020) indicam que os casos importados por meio da rede de transporte público foram um fator importante na dispersão de Sars-Cov-2. Hu et al. (2021) também concluíram que existe um alto risco de transmissão entre os passageiros do trem, sendo que os riscos variam de acordo com o tempo de viagem e a localização do assento. Harris (2020) demonstra que as linhas de metrô e ônibus semeiam maciçamente a Covid-19 na cidade de Nova York, diante da forte correlação positiva entre a distância das estações de transporte público e os domicílios com registro de casos de Covid-19. Em relação à restrição de mobilidade, Badr et al. (2020) encontraram uma forte correlação entre sua diminuição e a redução no crescimento de casos de Covid-19. Em contraste, o transporte privado oferece um ambiente relativamente seguro para as pessoas em comparação ao metrô e aos ônibus (LEIVA et al., 2020).

Estudos também investigaram possíveis associações entre Covid-19 e transporte público em grandes centros urbanos no Brasil. Em pesquisa realizada na cidade de São Paulo investigando 3.217 domicílios entre 18 e 20 de agosto de 2020, a prevalência total de Covid-19 foi de $11 \%$ (SÃO PAULO, 2020). Nessas pesquisas sorológicas, os pesquisadores usaram a coleta venosa e a separação do soro aplicando o teste imunocromatográfico IGM/IGG em adultos. Entre os usuários do transporte público, 11,3\% das pessoas foram infectadas com o vírus. A pesquisa mostra que as pessoas que trabalham em casa tiveram uma prevalência menor (4,4\%). Além disso, $16 \%$ das pessoas que vivem em domicílios com cinco moradores ou mais tiveram contato com a doença. Estudos anteriores mostram que a maioria das pessoas residentes em domicílios com cinco pessoas ou mais em São Paulo vive em periferias pobres e depende de serviços públicos, como transporte e saúde (LIBÂNIO, 2016). Rodrigues (2020) mostra que usuários de transporte público em São Paulo foram significativamente afetados pela Covid-19, dada a forte associação positiva entre bairros com elevado número de usuários e níveis de contaminação. Silva et al. (2020) também encontraram uma correlação espacial entre o uso do transporte público e os casos da doença em Recife. Em Belo Horizonte, Leiva, Sathler e Orrico Filho (2020) constataram que os casos de Covid-19 foram maiores nas áreas centrais, que concentram serviços públicos, como transporte. Em Belo Horizonte, como observamos em outras grandes cidades do Brasil, a maioria dos usuários de transporte vive em áreas periféricas e deve passar por áreas centrais, dada a topologia da rede de transporte público do município (LEIVA; SATHLER; ORRICO FILHO, 2020). Considerando a estrutura radiocêntrica do sistema de transporte em Belo Horizonte, esses resultados sugerem que imunizar os usuários do 
transporte público pode contribuir para diminuir a disseminação de Covid-19 advinda das áreas centrais em direção às periferias pobres.

Além disso, as pessoas de baixo nível socioeconômico usam principalmente ônibus e metrô nas grandes cidades brasileiras. Em 2011, a renda per capita familiar de $71,81 \%$ dos usuários de transporte público no Brasil era de até um salário mínimo (IPEA, 2012), enquanto essa proporção cai para 50,00\% entre os usuários de automóveis. De acordo com Patel et al. (2020), a população pobre tem maior probabilidade de morar em domicílios superlotados, o que diminui o cumprimento do distanciamento social, bem como de trabalhar em ocupações que não permitem atividades de home office. Além disso, a pobreza está associada a níveis mais baixos de conhecimento pessoal sobre pandemias (TIMMERMANN, 2020), trabalho precário (MENÉNDEZ et al., 2007), poupança limitada (SÁNCHEZ-PÁRAMO, 2020), alta dependência de serviços públicos e alta incidência de comorbidades (MENDENHALL et al., 2017). A integração de dados epidemiológicos e socioeconômicos no Brasil indica que pessoas de baixo nível socioeconômico foram fortemente afetadas pela Covid-19 (RODRIGUES, 2020).

Segundo o IPEA (2012), 65\% da população brasileira utiliza o transporte público como principal meio de locomoção. Diante desse alto percentual, as políticas de vacinação devem ter como foco os indivíduos que fazem uso frequente e que dependem do transporte público para o deslocamento diário. Segundo a ANTP (2020), 60 milhões de pessoas utilizavam o transporte público no Brasil diariamente em 2018, o que correspondia a $28,64 \%$ da população total do país.

TABELA 1

Viagens pendentes e tempo médio de viagem, por meio de transporte Belo Horizonte, Rio de Janeiro e São Paulo - 2012-2017

\begin{tabular}{|c|c|c|c|c|c|c|}
\hline \multicolumn{2}{|c|}{ Municípios } & \multirow{2}{*}{$\begin{array}{c}\begin{array}{c}\text { Transporte } \\
\text { público }\end{array} \\
855.769\end{array}$} & \multirow{2}{*}{$\begin{array}{c}\text { Individual } \\
962.984\end{array}$} & \multirow{2}{*}{$\begin{array}{c}\text { Não } \\
\text { motorizado } \\
630.385\end{array}$} & \multirow{2}{*}{$\begin{array}{l}\text { Outros } \\
155.795\end{array}$} & \multirow{2}{*}{$\frac{\text { Total }}{2.604 .933}$} \\
\hline & Número de viagens & & & & & \\
\hline \multirow[t]{3}{*}{ Belo Horizonte } & Viagens (\%) & 33,0 & 37,0 & 24,0 & 6,0 & 100,0 \\
\hline & Tempo & $01: 14: 44$ & $00: 34: 26$ & $00: 17: 42$ & 00:52:38 & $00: 42: 30$ \\
\hline & Número de viagens & 2.977 .536 & 762.886 & 650.732 & 23.904 & 4.415 .057 \\
\hline \multirow[t]{3}{*}{ Rio de Janeiro } & Viagens (\%) & 67,0 & 17,0 & 15,0 & 1,0 & 100,0 \\
\hline & Tempo & 01:01:18 & $00: 45: 26$ & $00: 17: 25$ & 01:04:34 & 00:50:34 \\
\hline & Número de viagens & 3.867 .676 & 2.978 .962 & 1.944 .633 & & 8.791 .271 \\
\hline \multirow[t]{2}{*}{ São Paulo } & Viagens (\%) & 44,0 & 34,0 & 22,0 & - & 100,0 \\
\hline & Tempo & 01:06:17 & $00: 30: 23$ & 00:11:30 & & $00: 39: 53$ \\
\hline
\end{tabular}

Fonte: Pesquisa Origem Destino de Belo Horizonte (2012); Pesquisa Origem Destino de Rio de Janeiro (2012); Pesquisa Origem Destino de São Paulo (2017).

Dados das regiões metropolitanas de Belo Horizonte, Rio de Janeiro e São Paulo sobre número total de viagens, percentual de viagens e tempo de deslocamento de acordo com o modo de transporte (público, individual, não motorizado e outros) revelam informações importantes sobre os fluxos de deslocamento nos três maiores e mais dinâmicos centros 
urbanos do Brasil. Essas três cidades têm um volume significativo de viagens diárias (15,8 milhões no total), com destaque para São Paulo $(8,79$ milhões) e Rio de Janeiro $(4,46$ milhões). Além disso, o tempo médio de viagem dos usuários do transporte público é elevado nas três cidades, ultrapassando uma hora, o que aumenta a exposição ao risco de contaminação pela Covid-19 durante as viagens. 0 Rio de Janeiro tem o maior percentual de usuários de transporte público (67\%), enquanto apenas $17 \%$ dos passageiros usam transporte particular individual.

De acordo com a Tabela 2, a maioria dos usuários do transporte público nessas três metrópoles ganha menos de dois salários mínimos (54\% dos usuários no Rio de Janeiro, $68 \%$ em São Paulo e 78\% em Belo Horizonte). Em contrapartida, o percentual de usuários de transporte particular individual com renda inferior a dois salários mínimos é significativamente menor em Belo Horizonte (44\%), Rio de Janeiro (26\%) e São Paulo (34\%) quando comparados aos valores apresentados pelos usuários do transporte público.

TABELA 2

Viajantes, por lasses de renda, segundo modos de transporte Belo Horizonte, Rio de Janeiro e São Paulo - 2012-2017

Em porcentagem

\begin{tabular}{|c|c|c|c|c|c|}
\hline & Municípios & \multicolumn{4}{|c|}{ Renda } \\
\hline \multirow{2}{*}{ Belo Horizonte } & Transporte público & 78,0 & 20,0 & 2,0 & 100,0 \\
\hline & Individual & 44,0 & 43,0 & 13,0 & 100,0 \\
\hline \multirow{3}{*}{ Rio de Janeiro } & Transporte público & 54,0 & 32,0 & 14,0 & 100,0 \\
\hline & Individual & 26,0 & 36,0 & 38,0 & 100,0 \\
\hline & Não motorizado & 70,0 & 18,0 & 12,0 & 100,0 \\
\hline \multirow{2}{*}{ São Paulo } & Não motorizado & 72,0 & 24,0 & 4,0 & 100,0 \\
\hline & Outros & - & - & - & - \\
\hline
\end{tabular}

Fonte: Pesquisa Origem Destino de Belo Horizonte (2012); Pesquisa Origem Destino de Rio de Janeiro (2012); Pesquisa Origem Destino de São Paulo (2017).

Esses dados sugerem que os formuladores de políticas públicas devem criar estratégias que protejam os usuários frequentes de transporte público da Covid-19. Nas maiores regiões metropolitanas, o transporte público é o único meio viável de acesso a empregos, educação e serviços para a maioria dos 11,4 milhões de habitantes que vivem em favelas no Brasil (IBGE, 2011). Embora grande parte da população suburbana em cidades extensas do mundo desenvolvido tenha acesso a carros, pessoas de baixo nível socioeconômico que vivem em subúrbios de grandes cidades brasileiras geralmente enfrentam alta exposição à Covid-19 no transporte público, o que desafia as políticas de reabertura econômica durante a pandemia. 
A ineficiência do sistema de transporte e as falhas significativas na estrutura de financiamento do transporte público no Brasil estão provavelmente associadas às altas taxas de disseminação de Covid-19 entre os funcionários e usuários de ônibus e metrô. Em grandes cidades espraiadas do país, o transporte público geralmente conecta centralidades urbanas a periferias distantes por meio de redes radiocêntricas dominadas pela alta competição entre ônibus e carros (ALVARENGA PEREIRA COSTA; TEIXEIRA, 2014; MARX et al., 2019). A forma urbana e a estrutura de transporte de São Paulo, Belo Horizonte e Rio de Janeiro afetam os níveis de congestionamento e o tempo médio de viagem, o que aumenta a ineficiência econômica para a manutenção do sistema (FERRAZ; TORRES, 2004). Além disso, a maior parte dos custos de viagem está incluída no preço da passagem no transporte público brasileiro (MARX et al., 2019). A falta de mecanismos inteligentes de financiamento para o sistema de transporte e a baixa viabilidade econômica das rotas planejadas em áreas de baixa densidade populacional aumentam a vulnerabilidade do sistema de transporte público em tempos de pandemia. Na maioria das grandes cidades brasileiras, a redução drástica na demanda de passageiros durante os primeiros meses da pandemia incentivou restrições à disponibilidade de transporte público. Portanto, à medida que a demanda aumenta, a disponibilidade deve crescer na mesma proporção, evitando aglomerações desnecessárias dentro de ônibus, metrôs e estações de espera.

Diante disso, pesquisadores e jornalistas têm investigado os possíveis cenários do mundo pós-pandêmico no setor de transportes. A percepção negativa da opinião pública sobre o transporte público como um meio de transporte de maior risco do ponto de vista da saúde pode afetar o ritmo de recuperação da demanda perdida (BAZANI, 2020; COLOMBO et al., 2020; PULLANO et al., 2020; TIRACHINI; CATS , 2020; XAVIER, 2020).

Dada a centralidade do transporte público nas discussões sobre cidades e Covid-19, a Associação Internacional de Transporte Público (UITP) solicitou à União Europeia que incluísse políticas voltadas ao transporte público urbano em seu plano de recuperação (XAVIER, 2020). Barcelos e Blank (2020) indicam que as ações realizadas durante a pandemia, como o reforço da limpeza dos espaços e a programação das atividades comerciais, devem contemplar a implementação de políticas que visem a sustentabilidade econômica do transporte público. Portanto, os autores argumentam que expandir a integração tarifária e formas alternativas de pagamento podem melhorar os indicadores operacionais em grandes centros urbanos. Na nossa opinião, a vacinação entre usuários de transporte público também pode ajudar as cidades a reduzirem as lacunas econômicas causadas pela diminuição do deslocamento diário no sistema de transporte público durante a pandemia de Covid-19.

Priorizar os usuários do transporte público na vacinação pode passar uma massagem relevante para a população: o transporte público é uma ferramenta importante para a criação de cidades democráticas e justiça social. Ao mesmo tempo, essa política será eficaz ao imunizar um grupo populacional com maior probabilidade de ser contaminado (durante o transporte ou nos espaços associados ao transporte) em comparação com usuários de transporte privado ou pessoas que trabalham em casa. A imunização protegerá não apenas 
os usuários frequentes do transporte público no Brasil, mas também outros residentes que não se deslocam diariamente, como a maioria da população idosa. A maior parte dos usuários de transporte público no Brasil apresenta vulnerabilidades associadas à pobreza e à desigualdade e depende exclusivamente do sistema público de saúde (PEREIRA et al., 2020). Portanto, priorizar a vacina entre os usuários pode reduzir a demanda nos hospitais públicos, economizar recursos públicos e reduzir significativamente os óbitos, uma vez que a mortalidade por Covid-19 no Brasil é maior entre as pessoas de baixo nível socioeconômico. Com a reabertura de grande parte das atividades econômicas do país e a intensificação do deslocamento envolvendo centro-periferia, a imunização dos usuários do transporte coletivo pode aumentar a eficiência das políticas de setorização do controle epidemiológico da doença, com foco em bairros ou regiões específicas da cidade. Os usuários contaminados do transporte público que fazem viagens longas, potencialmente, podem disseminar a doença de forma abrangente nas grandes cidades, contribuindo para a existência de novos surtos em áreas já controladas. Portanto, a imunização de pessoas que utilizam com frequência o transporte público nas grandes cidades pode contribuir para diminuir os níveis de contaminação e óbitos, evitando novas ondas de contaminação.

As grandes cidades brasileiras costumam apresentar melhores mecanismos para identificar os usuários frequentes do transporte público. Por exemplo, em Belo Horizonte, os usuários cadastrados possuem o cartão BHBus, que facilita o pagamento. Este cartão inclui a identificação (nome e foto) de parte significativa dos usuários frequentes do transporte público da cidade. Essas informações estão disponíveis no cadastro do sistema, mas também poderiam ser conferidas com os empregadores, que informariam à prefeitura a quantidade de funcionários beneficiados com o vale-transporte. 0 principal desafio é incluir a população informal nesta campanha, embora parte desse público também use o cartão BHBus.

Essa discussão pode subsidiar políticas públicas não apenas no Brasil, mas também em outros países em desenvolvimento onde a Covid-19 tem ampla difusão. Considerando todos os argumentos apresentados, o ritmo da vacinação no Brasil e também as limitações para implementar nossas ideias (como a identificação deste grupo-alvo), acreditamos que as autoridades brasileiras (especialmente as autoridades locais) devem considerar os usuários frequentes de transporte público na vacinação após concluírem a imunização do grupo prioritário definido pelo Plano Nacional. Priorizar os usuários do transporte público parece mais apropriado para as grandes e médias cidades, onde o transporte público é muito relevante nos deslocamentos diários. Além disso, as grandes cidades costumam ter mais ferramentas para identificar os usuários do transporte público, como o sistema BHBus de Belo Horizonte. Com base nas singularidades locais, cada cidade pode definir a forma como considera nossas sugestões. Existe mais de uma possiblidade, a saber: usuários de transporte público entre 18 e 59 anos podem ter acesso à vacina primeiro; após completar a vacinação entre os grupos etários adultos mais avançados, também é possível estabelecer esse tipo de prioridade apenas entre os adultos mais jovens (como 18 a 40 anos), que apresentam menores riscos associados ao envelhecimento; mesmo se as 
autoridades considerarem primeiramente os usuários de transporte na vacinação dentro de grupos de idade específicos, quando a idade é o principal critério, pode ser positivo em áreas urbanas maiores. Portanto, não há uma receita única para considerar nossas sugestões de priorização de usuários de transporte público em campanhas de vacinação no Brasil (ou mesmo em outras cidades do mundo).

Em suma, considerando o recente surgimento de vacinas eficazes contra a Covid-19 e os escassos recursos para atender imediatamente à maior parte da população mundial, as sociedades precisam definir a ordem em que os grupos de cidadãos terão acesso às novas vacinas. Conforme sugerido pela OMS (WHO, 2020), as campanhas de vacinação devem priorizar a imunização de indivíduos vulneráveis e trabalhadores de serviços essenciais, reduzindo as perdas humanas e minimizando os danos sociais e econômicos. No Brasil, os usuários de transporte público nas grandes cidades apresentam altos níveis de vulnerabilidade, em função de fatores relacionados à configuração espacial, à organização dos sistemas de transporte e ao alto percentual de pessoas de baixo nível socioeconômico em grandes periferias que dependem exclusivamente do transporte público para acesso a empregos e serviços básicos. A imunização dos usuários do transporte público pode produzir efeitos práticos relevantes no combate à Covid-19 no Brasil, como economia de recursos públicos, redução do número de óbitos e maior eficiência no controle setorizado da doença nas cidades. Portanto, sugerimos que os formuladores de políticas devem considerar os usuários frequentes de transporte público das grandes cidades brasileiras como um grupo-alvo nas campanhas de vacinação, dando a este grupo um certo nível de prioridade com base no mapeamento de risco adequado em nível local, especialmente nos grandes centros urbanos.

\section{Agradecimentos}

Os autores agradecem o Dr. Michael J. Passow (Columbia University) pelas sugestões que contribuíram para tornar o texto mais claro.

\section{Referências}

ALVARENGA PEREIRA COSTA, S. de; TEIXEIRA, M. C. V. The study of urban form in Brazil. Urban Morphology, v. 18, n. 2, p. 119-127, 2014.

ANTP - Associação Nacional de Transportes Públicos. Sistema de Informações da Mobilidade Urbana da Associação Nacional de Transportes Público - SIMOB/ANTP. Relatório geral 2018. São Paulo, 2020. Disponível em: http://files.antp.org.br/simob/sistema-de-informacoes-damobilidade--simob--2018.pdf. Acesso: 12 mar. 2021.

BADR, H. S. et al. Association between mobility patterns and COVID-19 transmission in the USA: a mathematical modelling study. The Lancet Infectious Diseases, v. 20, n. 11, p. 1247-1257, 2020.

BARCELOS, M. M.; BLANK, C. Transporte coletivo e transmissão da Covid-19: o que dizem os estudos. WRI Brasil, 1 out. 2020. Disponivel em: https://wribrasil.org.br/pt/blog/transportecoletivo-e-transmissao-da-covid-19-o-que-dizemosestudos. Acesso em: 23 mar. 2021. 
BAZANI, A. Ônibus tem atraído mais passageiros que metrô em Nova lorque e é visto como solução na retomada em meio à pandemia. Portal Diário do Transporte, 12 jul. 2020. Disponível em: https://diariodotransporte.com.br/2020/07/12/onibustematraido-mais-passageiros-quemetro-em-nova-iorque-e-e-visto-como-solucao-naretomada-em-meio-a-pandemia/. Acesso em: 23 mar. 2021

BRASIL. Ministério da Saúde. Boletim Epidemiológico do Ministério da Saúde. Brasília, 2021. Disponivel em: https://coronavirus.saude.gov.br/boletins-epidemiologicos.

COLOMBO, S. et al. O impacto da pandemia do Covid-19 no transporte coletivo no município de Cuiabá - Mato Grosso. In: XVII CONGRESSO RIO DE TRANSPORTES. Anais [...]. Rio de Janeiro, 2020.

DIAS, R. Especialistas criticam pontos do plano nacional de vacinação do governo. Estado de Minas, $16 / 12 / 2020$.

ECDC - European Centre for Disease Prevention and Control. Considerations for infection prevention and control measures on public transport in the context of COVID-19. Stockholm, 2020. Disponivel em: https://www.ecdc.europa.eu/sites/default/files/documents/COVID-19public-transport-29-April-2020.pdf.

FERRAZ, A. C. C. P.; TORRES, I. G. E. Transporte público urbano. São Carlos: Rima, 2004.

HARRIS, J. The subways seeded the massive coronavirus epidemic in New York City. Cambridge: National Bureau of Economic Research, 2020 (NBER Working Paper, n. 27021).

$\mathrm{HU}, \mathrm{M}$. et al. The risk of COVID-19 transmission in train passengers: na epidemiological and modelling study. Clinical Infectious Diseases, v. 72, n. 4, p. 604-610, 2021. Doi:10.1093/cid/ ciaa1057/5877944.

IBGE - Instituto Brasileiro de Geografia e Estatística. Censo Demográfico do Brasil 2010. Rio de Janeiro, 2011.

IPEA - Instituto de Pesquisa Econômica Aplicada. Sistema de Indicadores de Percepção Social (SIPS). Mobilidade urbana. Brasília, 2012. Disponível em: https://www.ipea.gov.br/portal/index. php?option=com_content\&view=article\&id=24443\& catid=120\&ltemid=2. Acesso: 12 mar. 2021.

LEIVA, G. et al. Transport and land use: public transport stragies to make cities more resilient to COVID-19. 2020.

LEIVA, G.; SATHLER, D.; ORRICO FILHO, R. Estrutura urbana e mobilidade populacional: implicações para o distanciamento social e disseminação da Covid-19. Revista Brasileira de Estudos de População, v. 37, 2020.

LIBÂNIO, C. de A. O fim das favelas? Planejamento, participação e remoção de famílias em Belo Horizonte. Cadernos Metrópole, v. 18, n. 37, p. 765-784, 2016.

MARX, R.; MAGALHÃES, P. S. de; LARA, F. F. de. Low-cost bus business models and the case of Brazil. Revista de Gestão, v. 27, n. 1, p. 100-115, 2019.

MATEUS, A. L. P. et al. Effectiveness of travel restrictions in the rapid containment of human influenza: a systematic review. Bulletin of the World Health Organization, v. 92, p. 868-880D, 2014. DOI: 10.2471/BLT.14.135590.

MENDENHALL, E. et al. Non-communicable disease syndemics: poverty, depression, and diabetes among low-income populations. The Lancet, v. 389, n. 10072, p. 951-963, 2017.

MENÉNDEZ, M. et al. Is precarious employment more damaging to women's health than men's? Social Science and Medicine, v. 64, n. 4, p. 776-781, 2007.

PARDO, C. F. et al. COVID-19 and public transport: an overview and recommendations applicable to Latin America. Infectio, v. 25, n. 3, p. 182-188, 2021. 
PATEL, J. A. et al. Poverty, inequality and COVID-19: the forgotten vulnerable. Public Health, v. 183, p. 110-111, January 2020.

PEREIRA, R. H. M. et al. Desigualdades socioespaciais de acesso a oportunidades nas cidades brasileiras - 2019. Brasília: Ipea, 2020. (Texto para Discussão, 2535).

PULLANO, G. et al. Population mobility reductions during COVID-19 epidemic in France under lockdown. medRxiv, June 2020.

RODRIGUES, A. Mortes por covid-19 têm mais relação com autônomos, donas de casa e transporte público. Valor Econômico, 09/08/2020.

SÁNCHEZ-PÁRAMO, C. COVID-19 will hit the poor hardest. Here's what we can do about it. World Bank Blogs, April 23, 2020.

SAO PAULO (Município). Inquérito sorológico para Sars-COV-2. Fase 4. São Paulo, 2020.

SILVA, C. F. A. da et al. Análise da correlação espacial entre os usuários de sistemas de transporte público e os casos de Covid-19: um estudo de caso para Recife (PE). Revista Brasileira de Gestão e Desenvolvimento Regional, v. 16, n. 4, dez. 2020. Disponível em: https://www.rbgdr.net/ revista/index.php/rbgdr/article/view/5989. Acesso em: 24 fev. 2021.

THANH LE, T. et al. The COVID-19 vaccine development landscape. Nature Reviews. Drug Discovery, v. 19, n. 5, p. 305-306, 2020.

TIMMERMANN, C. Epistemic ignorance, poverty and the COVID-19 pandemic. Asian Bioethics Review, v. 12, p. 519-527, 2020.

TIRACHINI, A.; CATS, O. COVID-19 and public transportation: current assessment, prospects, and research needs. Journal of Public Transportation, v. 22, n. 1, 2020. DOI: https://doi. org/10.5038/2375-0901.22.1.1. Disponível em: https://scholarcommons.usf.edu/jpt/vol22/ iss $1 / 1$.

WHO - World Health Orzanization. Coronavirus disease (COVID-2019) situation reports. [s.I], 2020. Disponivel em: https://www.who.int/emergencies/diseases/novel-coronavirus-2019/ situation-reports.

XAVIER, B. O. Transporte público por ônibus no Brasil e a Covid-19: rumo ao colapso dos sistemas? In: CONGRESSO DE PESQUISA E ENSINO EM TRANSPORTES. 34. Anais [...]. Associação Nacional de Pesquisa e Ensino em Transportes, 2020. p. 282-293.

ZHENG, R.; XU, Y.; WANG, W.; NING, G.; BI, Y. Spatial transmission of COVID-19 via public and private transportation in China. Travel Medicine and Infectious Disease, Mar. 2020. DOI: 10.1016/j. tmaid.2020.101626.

\section{Sobre os autores}

Douglas Sathler é doutor em Demografia pela Universidade Federal de Minas Gerais (UFMG) e geógrafo pela UFMG. Professor da Universidade Federal dos Vales do Jequitinhonha e Mucuri (UFVJM).

Guilherme de Castro Leiva é doutor em Demografia pela Universidade Federal de Minas Gerais (UFMG), mestre em Arquitetura e Urbanismo pela UFMG e arquiteto e urbanista pela Pontifícia Universidade Católica de Minas Gerais (PUC Minas). Professor do Centro Federal de Educação Tecnológica de Minas Gerais (Cefet-MG). 


\title{
Endereço para correspondência
}

\author{
Douglas Sathler \\ Faculdade Interdisciplinar de Humanidades, campus JK \\ Rodovia MGT 367 - km 583, n. 5.000, Alto da Jacuba \\ 39100-000 - Diamantina-MG, Brasil \\ Guilherme de Castro Leiva \\ Centro Federal de Educação Tecnológica de Minas Gerais, campus Nova Suíça \\ Departamento de Engenharia de Transportes, sala 101 \\ Av. Amazonas, 5.253, Nova Suiça \\ 30421-169 - Belo Horizonte-MG, Brasil
}

\begin{abstract}
Priority for future COVID-19 vaccinations in Brazil: should public transport users be a target group?
\end{abstract}

Considering the recent emergence of effective vaccines against COVID-19 and the scarce resources to assist most of the world population immediately, societies need to define the order in which groups of citizens will get access to new vaccines. Vaccination campaigns should prioritize the immunization of vulnerable individuals and people tasked with helping others, thus reducing human losses and minimizing social and economic damage. In Brazil, public transport users in large cities present high levels of vulnerability, due to factors related to the spatial configuration, the organization of transport systems and the high percentage of people of low socio-economic status within large suburbs that depend exclusively on public transport to access jobs and basic services. Immunizing public transport users can produce relevant practical effects in combating COVID-19 in Brazil, such as saving public resources, reducing the number of deaths and achieving greater efficiency in the sectorized control of the disease within cities. Therefore, we suggest that policymakers should consider the frequent users of public transport from large Brazilian cities as a target group in vaccination campaigns, affording this group some priority based on adequate risk mapping at the local level.

Keywords: Vaccine. COVID-19. Public transport. Target group.

\section{Resumen}

Prioridad para futuras vacunaciones contra la covid-19 en Brasil: los usuarios de transporte deben ser un grupo objetivo?

Teniendo en cuenta la reciente aparición de vacunas eficaces contra la covid-19 y los escasos recursos para atender de inmediato a la mayoría de la población mundial, las sociedades deben definir el orden en el que los grupos de ciudadanos tendrán acceso a las nuevas vacunas. Las campañas de vacunación deben priorizar la inmunización de personas vulnerables y personas encargadas de ayudar a otras para reducir las pérdidas humanas y minimizar los daños sociales y económicos. En Brasil, los usuarios del transporte público en las grandes ciudades presentan altos niveles de vulnerabilidad, dados los factores relacionados con la configuración 
espacial, la organización de los sistemas de transporte y el alto porcentaje de personas de nivel socioeconómico bajo dentro de los grandes suburbios que dependen exclusivamente del transporte público para acceder a empleos y servicios básicos. La vacunación de los usuarios del transporte público puede producir efectos prácticos relevantes en el combate a la covid-19 en Brasil, como el ahorro de recursos públicos, la reducción del número de muertes y una mayor eficiencia en el control sectorizado de la enfermedad en las ciudades. Por lo tanto, sugerimos que los formuladores de políticas consideren a los usuarios frecuentes del transporte público de las grandes ciudades brasileñas como un grupo objetivo en las campañas de vacunación y le den a este grupo cierto nivel de prioridad basado en un adecuado mapeo de riesgos a nivel local.

Palabras clave: Vacuna. Covid-19. Transporte público. Grupo objetivo.

Recebido para publicação em 15/02/2021 Aceito para publicação em 10/06/2021 